Research Article

Open Access

\title{
Epithelial Mesenchymal Transition enhances the Chemosensitivity of Triple Negative Breast Cancer Cells to JQ1
}

\author{
Yuan You' ${ }^{1}$ and Michael J Rossi2 ${ }^{*}$ \\ IResearch Assistant, Department of Biology and Environmental Sciences, University of New Haven, West Haven, USA \\ ${ }^{2}$ Associate Dean and Professor, Department of Biology and Environmental Sciences, University of New Haven, West Haven, USA
}

\section{Article Info}

*Corresponding author:

Michael J Rossi

Associate Dean

University of New Haven

300 Boston Post Road, West Haven, CT 06516

USA

E-mail: MRossi@newhaven.edu

Received: October 25, 2017

Accepted: December 6, 2017

Published: December 12, 2017

Citation: You Y, Rossi MJ. Epithelial Mesenchymal Transition enhances the Chemosensitivity of Triple Negative Breast Cancer Cells to JQ1. Madridge J Oncogenesis. 2017; 1(1): 12-20.

doi: $10.18689 / \mathrm{mjo}-1000103$

Copyright: (c) 2017 The Author(s). This work is licensed under a Creative Commons Attribution 4.0 International License, which permits unrestricted use, distribution, and reproduction in any medium, provided the original work is properly cited.

Published by Madridge Publishers

\begin{abstract}
Triple negative breast cancers (TNBC) are particularly aggressive and more likely to recur than other subtypes of breast cancer. Bromodomain and extra terminal domain (BET) inhibitors have shown efficacy in several cancer models by inhibiting BET function. JQ1, a specific Bromodomain inhibitor, functions as a competitive inhibitor, with $I C_{50}$ values from $92-112 \mathrm{nM}$.
\end{abstract}

\section{Objectives}

To study the inhibitory effect of JQ1 to TNBC cells in 2D and 3D culture systems.

\section{Methods}

In this study, triple negative mesenchymal-like cell lines (MDA-MB-231, Hs578T) and basal-like cell line (Hcc1143) were used to examine the inhibitory effect of JQ1. A 3D culture system was used to potentially activate an epithelial-mesenchymal transition (EMT) in these lines. MTT assays and 3D apoptosis assays were performed to determine the inhibitory effect of JQ1 to the TNBC cells.

\section{Results}

Markers for EMT were expressed when Hcc1143 cells were cultured in the 3D culture, but this was not seen for MDA-MB-231 and Hs578T cells. However all three cell lines had significantly higher bromodomain protein Brd4 mRNA expression in 3D cultures and increased the expression of bromodomain proteins compared to 2D cultures. Viability assays determined that the $\mathrm{IC}_{50}$ for JQ1 in the $2 \mathrm{D}$ system ranged from 30 to $170 \mathrm{nM}$. In the $3 \mathrm{D}$ system the $I C_{50}$ ranged from 10 to $80 \mathrm{nM}$. Hcc 1143 cells were more sensitive to JQ1 in the 3D system than in a 2D system.

\section{Conclusion}

JQ1 inhibited the growth of TNBC cells in both systems and shows potential for further studies of triple negative breast cancer.

Keywords: Triple Negative Breast Cancer; Epithelial-Mesenchymal Transition; Bromodomain Protein; JQ1.

\section{Introduction}

Breast cancer is the second most common cancer among American women, about $12 \%$ of women in the US will develop invasive breast cancer during their lives [1]. In the United States, The American Cancer Society estimated 255,180 new cases of invasive breast cancer and 40,610 deaths in 2017 [1]. Breast cancer is classified based on 
molecular properties including the expression of estrogen receptor $(E R \alpha)$, progesterone receptor $(\mathrm{PgR})$ and human epidermal growth factor receptor 2 (HER2) [2]. Triple-negative breast cancers (TNBC) do not express the genes for ER, PgR or Her2. TNBC occurs in $10-20 \%$ of diagnosed breast cancers, but leads to $25 \%$ of all breast cancer deaths [3]. Depending on the stage of its diagnosis, triple-negative breast cancer can be particularly aggressive, and more likely to recur than other subtypes of breast cancer [2]. While triple-negative tumors generally do not respond to receptor targeted treatments, TNBC is typically responsive to initial chemotherapy [2].

Alsarraj and Hunter (2011) determined that bromodomaincontaining protein 4 (BRD4) functions as an inherited susceptibility gene for breast cancer progression and metastasis. They hypothesized that it could be a potential therapeutic target for TNBCs [4].

BRD4 is one member of Bromodomain and extraterminal domain family (BET), including BRD2, BRD3, and BRDT. BET protein domains were originally found as proteins associated with chromatin organization and nearly all nuclear histone acetyltransferases [5]. Proteins of the BET family are key mediators for the assembly of the positive transcription elongation factor $b$ complex (P-TEFb), an event required for the initiation of transcription elongation [6]. The P-TEFb core complex, composed of cyclin-dependent kinase- 9 and its activator cyclin T, functions to activate RNA polymerase II [7]. BET proteins are also required for maintaining a proper higher-order chromatin structure. Importantly, BET proteins have been shown to be implicated in cancer, where they can be expressed as malignant oncogenic fusion proteins together with nuclear protein in testis (NUT), resulting in very aggressive and poorly differentiated carcinomas that originate mainly from midline locations such as the head, neck, or mediastinum $[8,9]$.

JQ-1 is one of the bromodomain inhibitors specific for the BET family, competitively binding to the acetyl-lysine recognition pocket of BET bromodomains $[9,10]$. Inhibition of the BET bromodomain with JQ-1 demonstrated potent anticancer effects both in vitro and in vivo in several different hematopoietic cancers as well as NUT midline carcinoma $[11,12]$.

In humans, cells reside in an extracellular matrix (ECM) consisting of a complex 3D fibrous meshwork with a wide distribution of fibers and peptidoglycans that provide complex biochemical and physical signals [13]. Commonly, a 3D cell culture system provides the culture environments where cell are allowed to interact with their surrounding cells in all three dimensions. Additionally, each type of cell is embedded in a specific 3D microenvironment [13]. Therefore, regular 2D cell culture systems are inadequate representations of the real living microenvironment, which often makes them unreliable predictors of in vivo drug efficacy and toxicity [14].

The development of cancer is a multistep process and the activation of epithelial-mesenchymal transition (EMT) plays a important role in cell dissemination $[15,16]$. EMT has also been shown to occur in wound healing, in organ fibrosis, and in the initiation of metastasis for cancer progression [17]. Whether the BRD2, BRD3 and BRD4 in TNBCs cultured in 2D and $3 \mathrm{D}$ will have similar gene and protein expression is not clear. To gain insights into the bromodomain protein's function on cell growth, the gene expression and protein expression of the BRD family were measured in TNBCs cultured in $2 D$ and $3 D$ conditions. We were also curious if MET and EMT will occur when TNBCs were growing in 3D systems compared to 2D systems. To answer these questions, cell proliferation in the presence of JQ1 was studied to clarify whether JQ1 inhibits TNBC growth. Cell viability assays were performed to determine the JQ1 inhibition of TNBC cells in 2D and 3D. The hypothesis was the 3D culture system would provide a more representative system to determine the effect of JQ1 on TNBC growth and viability.

\section{Methods and Materials}

\section{Cell lines and culture conditions}

The cell line MDA-MB-231 (ATCC ${ }^{\circledR}$ HTB-26 $6^{\text {TM}}$ ) was cultured in DMEM-F12 medium supplemented with $10 \%(\mathrm{v} / \mathrm{v})$ fetal bovine serum (Serum Source International) and 1\% (v/v) L-Glutamine with antibiotics (Sigma, USA). The cell line Hs578T cells (ATCC ${ }^{\circledR}$ HTB-126 ${ }^{\mathrm{TM}}$ ) was cultured in DMEM medium supplemented with $10 \%(\mathrm{v} / \mathrm{v})$ fetal bovine serum and $1 \%(\mathrm{v} / \mathrm{v})$ L-Glutamine with antibiotics. The cell line HCC143 (ATCC ${ }^{\otimes}$ CRL$\left.2321^{\mathrm{TM}}\right)$ was cultured in RPMI-1260 medium supplemented with $10 \%(\mathrm{v} / \mathrm{v})$ fetal bovine serum and $1 \%(\mathrm{v} / \mathrm{v})$ L-Glutamine with antibiotics. The cultures was incubated at $37^{\circ} \mathrm{C}$ and $5 \%$ $\mathrm{CO}_{2}$, maintained in $75 \mathrm{~cm}^{2}$ polystyrene coated tissue culture flasks and passaged every 3 days at $70 \%$ confluence.

\section{D cell Culture conditions}

In 3D cell culture system, $3 \mathrm{ml}$ of $10 \%(\mathrm{~g} / \mathrm{v})$ gelatin (Type A, Sigma, USA) was incubated in $100 \mathrm{~mm}$ cell culture dishes (Corning, Sigma, USA) for 30 minutes until the gelatin became solid. Then $10^{6}$ cells were transferred and cultured with $6 \mathrm{ml}$ growth medium for 48 hours.

\section{Reverse transcription PCR}

RNA extractions from cells cultured in $2 \mathrm{D}$ and $3 \mathrm{D}$ were performed by using the Trizol Kit (Ambion ${ }^{\circledR}$, Life Technologies. USA). RT-PCR was performed using the Verso ${ }^{\mathrm{TM}}$ CDNA Kit (Thermo Scientific, AB-1453). cDNA was reverse transcribed from $1 \mu \mathrm{g}$ RNA. PCR amplification was done under the following conditions: $47^{\circ} \mathrm{C}$ for 1 hour for CDNA synthesis and $95^{\circ} \mathrm{C}$ for 2 minutes for inactivation and hold at $4^{\circ} \mathrm{C}$. The cDNA was diluted to $5 \mathrm{ng} / \mu \mathrm{l}$.

\section{Quantitative PCR}

Quantitative PCR was performed using a reaction mix (PCR Kappa Supermix, Thermo Fisher, USA) containing Tag DNA polymerase, Tris- $\mathrm{HCl}$, potassium chloride, magnesium chloride and deoxynucleoside triphosphates. In detail, $12.5 \mathrm{ng}$ of cDNA was mixed with $5 \mu$ l of Supermix (Thermo Fisher, USA) and $2.5 \mu$ of primer pairs ( $1 \mu \mathrm{M}$ forward primer: $1 \mu \mathrm{M}$ 
reverse primer). qPCR was performed in the Bio-Rad CFX96 ${ }^{\text {TM }}$ Real-Time System for 2 hours under the following conditions: $95^{\circ} \mathrm{C}$ for 15 minutes for initial melting; $94^{\circ} \mathrm{C}$ for 1 minute, $58^{\circ} \mathrm{C}$ for 1 minute, and $72^{\circ} \mathrm{C}$ for 1 minute for a total of 40 cycles; and $72^{\circ} \mathrm{C}$ for 10 minutes for final extension. Primers used are shown in Table 1.

Table 1. The primer sequences of BRD2, BRD3, BRD4 and GAPDH

\begin{tabular}{|l|l|l|l|}
\hline Common Name & Gene Name & Forward & Reverse \\
\hline BRD2 & BROMODOMAIN-CONTAINING PROTEIN 2 & $5^{\prime}$-TCCGTTATGCCAAGATGCCA-3' & 5'-GCCCGAAGCTGTTCCTGTAG-3' \\
\hline BRD3 & BROMODOMAIN-CONTAINING PROTEIN 3 & $5^{\prime}$-GTTCGCCTGGCCCTTCTAC -3' & 5'-ACTATGTCATCTGTGGGCTTGT-3' \\
\hline BRD4 & BROMODOMAIN-CONTAINING PROTEIN 4 & $5^{\prime}$-CCTCCAACCCTAACAAGCCC -3' & 5'-AGTAATCAGGGAGGTTCAGCTT-3' \\
\hline GAPDH & GLYCERALDEHYDE-3-PHOSPHATE DEHYDROGENASE & $5^{\prime}$-GAGTCAACGGATTTGGTCGT-3' & 5'-GACAAGCTTCCCGTTCTCAGCC-3' \\
\hline
\end{tabular}

RT-qPCR quantification

$\mathrm{Cq}$ values were measured by CFX Manager ${ }^{\mathrm{TM}}$ Software (Bio-Rad, USA). The average of $\mathrm{Cq}$ values of each gene was calculated using Excel software. The $\Delta \Delta \mathrm{Cq}$ method were performed to quantify the gene expression level, and GAPDH house keeping gene was used as controls for both $2 \mathrm{D}$ and $3 \mathrm{D}$ cultures.

\section{Protein Extraction}

$500 \mu$ l of ice cold $1 X$ cell lysis buffer (Cell Signaling, \#9803, USA) was added into the collected cells and then incubated for 30 minutes on ice. After the centrifuge for 10 minutes at $14,000 \mathrm{xg}$ at $4^{\circ} \mathrm{C}$, the supernatant were collected for use.

\section{Western Blot}

For western blots, 40 mug total protein extraction lysates were loaded in $7.5 \%$ SDS-polyacrylamide gel and transferred to PVDF membranes. The membranes were blocked for 1 hour with the $5 \%$ milk/PBST $\left(8 \mathrm{mM} \mathrm{Na}_{2} \mathrm{HPO}_{4}, 150 \mathrm{mM} \mathrm{NaCl}, 2 \mathrm{mM}\right.$ $\mathrm{KH}_{2} \mathrm{PO}_{4}, 3 \mathrm{mM} \mathrm{KCl}, 0.05 \%$ Tween $^{\circledR} 20, \mathrm{pH}$ 7.4) and then incubated with primary antibodies overnight separately: mouse anti-human BRD3 (2088C3a, Santa Cruz, USA, 1:1000); rabbit anti-human BRD4 (ab128874, Abcam, USA, 1:1000); rabbit anti-human Vimentin (5741, Cell Signaling, USA, 1:1000); rabbit anti-human YY1 (ab12132, Abcam, USA, 1:1000); rabbit anti-human Actin (ab8227, Abcam, USA, 1:2000) and mouse anti-human GAPDH (ab9483, Abcam, USA,1:10000). After incubation with the corresponding secondary antibody (goat anti-rabbit $\lg \mathrm{G}(\mathrm{H}+\mathrm{L})$ horseradish peroxidase (HRP) conjugated antibodies (Jackson ImmunoResearch Laboratories, Inc., USA)) or anti-mouse $\lg G(\mathrm{H}+\mathrm{L})$ horseradish peroxidase (HRP) conjugated antibodies (Jackson ImmunoResearch Laboratories, Inc., USA) at a dilution of 1:10,000 for 2 hours, membranes were finally incubated with a chemiluminescent reagent (Clarity ${ }^{\mathrm{TM}}$ Western ECL Blotting Substrate, Bio Rad, USA) for 3 minutes and the chemiluminescent blots were recorded by FluorChem ${ }^{\mathrm{TM}}$ E System (ProteinSimple, USA).

\section{Quantification Analysis of Western Blots}

Western images were subsequently imaged with the Fluor Chem $^{\text {TM }}$ E System using the white light conversion screen and the silver stain (visible stain) application. The band analysis tools of Alpha View ${ }^{\mathrm{TM}}$ software were used to select and determine the background-subtracted density of the bands in all the gels and blots. Quantification analysis of Brd3 and $\mathrm{Brd} 4$ protein expressions was performed by using GAPDH as control for total protein.

\section{D Cell Viability Assay}

MTT assays were performed to determine cell viability under the treatment of JQ1 in a 2D cell culture system. For this assay, $1 \times 10^{6}$ cells were transferred into each well of 48well plates with $500 \mu \mathrm{l}$ medium. Cells were treated with $1 \mu \mathrm{l}$ JQ-1 and Carboplatin at various final concentrations $(0 \mu \mathrm{M}$, $2.5 \mu \mathrm{M}, 5 \mu \mathrm{M}, 10 \mu \mathrm{M}, 20 \mu \mathrm{M}, 40 \mu \mathrm{M}, 80 \mu \mathrm{M}, 160 \mu \mathrm{M}, 320 \mu \mathrm{M}$ and $640 \mu \mathrm{M}$ ). After 3 days, $50 \mu \mathrm{l}$ of $5 \mathrm{mg} / \mathrm{ml} \mathrm{MTT}$ solution was directly added to all wells except the blank wells. After the incubation of 3 hours at $37^{\circ} \mathrm{C}$, the medium in each well was removed and $500 \mu \mathrm{l}$ of DMSO and $25 \mu \mathrm{l}$ of Sorensen's glycine buffer $(0.1 \mathrm{M}$ glycine, $0.1 \mathrm{M} \mathrm{NaCl}$ equilibrated to $\mathrm{pH} 10.5$ with $0.1 \mathrm{M} \mathrm{NaOH}$ ) was added into each well. With gentle shaking, color was allowed to develop for at least $10 \mathrm{~min}$ at room temperature, and then the absorbance at $570 \mathrm{~nm}$ was determined. The $\mathrm{ED}_{50}$ of JQ-1 and Carboplatin for each cell line were determined by analyzing the data of three experiments using the Prism 6 Program (GraphPad Software, Inc, USA).

\section{D Live Cell Imaging}

Effect of JQ1 on cell proliferation was investigated by recording time-lapse images of the cells for 3 days. $50 \mu \mathrm{l}$ of $10 \%$ gelatin were plated in the 96 well plate and incubated for 30 minutes. MDA-MB-231 cells, Hs578T cells and Hcc1143 cells $\left(6 \times 10^{3}\right)$ were seeded into 96 -well plates and allowed to attach for $24 \mathrm{hr}$. The media was replaced with fresh medium containing different concentrations of JQ1 $(0 \mu \mathrm{M}, 2.5 \mu \mathrm{M}, 5$ $\mu \mathrm{M}, 10 \mu \mathrm{M}, 20 \mu \mathrm{M}, 40 \mu \mathrm{M}, 80 \mu \mathrm{M}, 160 \mu \mathrm{M}, 320 \mu \mathrm{M}, 640 \mu \mathrm{M}$ and $1280 \mu \mathrm{M})$. The plate was imaged in phase using an IncuCyte ZOOM $^{\text {TM }}$ Kinetic Imaging System (Essen Bioscience) every 2 hours for 3 days. Inbuilt software was used for analysis of the images to generate confluence data.

\section{D Cell Viability Assay}

Cell Titer-Glo ${ }^{\circledR}$ 3D Cell Viability Assay (Promega, USA) were performed to determine cell viability under the treatment of JQ1 at 3D cell culture system. For this assay, $50 \mu \mathrm{l}$ of $10 \%$ 
gelatin were plated into each well of the 96 well plate and incubated for 30 minutes. And then 6,000 cells were transferred into each well of 48 -well plates with $100 \mu \mathrm{l}$ medium. After 24 hours' culture, cells were treated with $1 \mu \mathrm{l}$ of JQ-1 at various final concentrations $(0 \mu \mathrm{M}, 2.5 \mu \mathrm{M}, 5 \mu \mathrm{M}, 10$ $\mu \mathrm{M}, 20 \mu \mathrm{M}, 40 \mu \mathrm{M}, 80 \mu \mathrm{M}, 160 \mu \mathrm{M}, 320 \mu \mathrm{M}$ and $640 \mu \mathrm{M}$ and $1280 \mu \mathrm{M})$. After 3 days, 3D cell viability assay were performed. In briefly, Cell Titer-Glo ${ }^{\circledR} 3 \mathrm{D}$ Reagent were thaw at $4^{\circ} \mathrm{C}$ overnight and placed in a $22^{\circ} \mathrm{C}$ water bath for 30 minutes prior to use. In 96 well plate, 50 ul of media was carefully took away from each well. $100 \mathrm{ul}$ of 3D Reagent were added into the contents and incubated at room temperature for 30 minutes. Luminescence measurements were recorded by reading all wavelengths with SpectraMax Plus 384 Microplate Reader (Molecular Devices, California, USA). The IC ${ }_{50}$ of JQ-1 for each cell line were determined by analyzing the data of

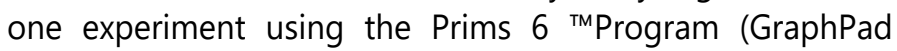
Software, Inc, USA).

\section{Statistical analysis}

The differences between two groups $(n=3)$ were determined with Mann-Whitney test by using Graph Pad Prism 6 statistical software (La Jolla, CA). A p-value of $\leq 0.05$ was considered as significant and data is presented as mean \pm standard deviation of the mean.

\section{Results}

\section{Gene expression of bromodomain proteins were induced in 3D cultures}

A comparison of $\mathrm{Cq}$ values of BRD2, BRD3 and BRD4 gene expression from $2 D$ and $3 D$ cultures determined the effect of the 3D cell culture on the gene expression of BET family transcripts. GAPDH was used as control in this qPCR assay. All three TNBC cell lines had significantly higher BRD4 expression in 3D than in 2D at 24 hours (Figure 1). However, only Hcc1143 had significantly higher BRD2 and BRD3 expression in 3D compared to 2D cultures at 24 hours.

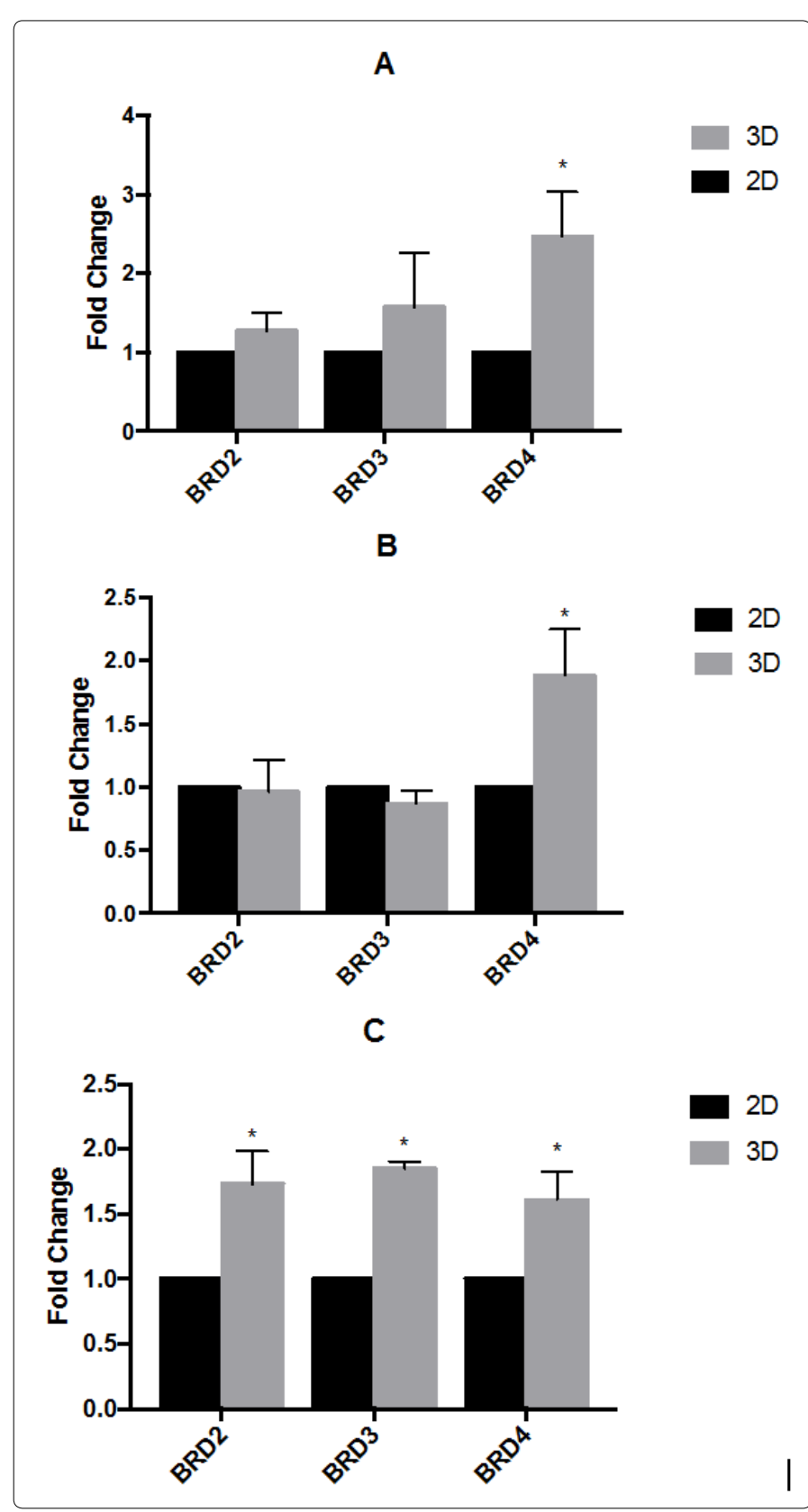

Figure 1. Gene expression of BRD2, BRD3 and BRD4 in TNBC cell lines

QPCR was performed using GAPDH as control. Gene expression of BRD2, BRD3 and BRD4. BRD4, determined by the $\triangle \triangle C$ q method, was significantly higher in $3 \mathrm{D}$ cultures for (A) MDA_MB-231, (B) Hs578T, and (C) Hcc1143 cells than in $2 D$ at 24 hours. BRD2 and BRD3 expression was not significantly changed for MDA-MB-231 or Hs578T cells, but they were significantly higher for Hcc1143 cells in 3D cultures.

\section{Brd3 and Brd4 were induced in 3D cultures}

BET Proteins Brd3 and Brd4 were expressed in all three TNBC cell lines (Figure 2A). Quantification of band intensity determined that MDA-MB-231 had $80 \%$ increase in Brd3 expression in 3D culture and Hcc1143 had three times expressions of $\mathrm{Brd} 3$ and $\mathrm{Brd} 4$ at 3D culture compared to 2D (figure 2B). Hs578T did not have significantly different Brd3 and $\mathrm{Brd} 4$ expression when they were cultured under the two conditions (Figure 2B). 
A
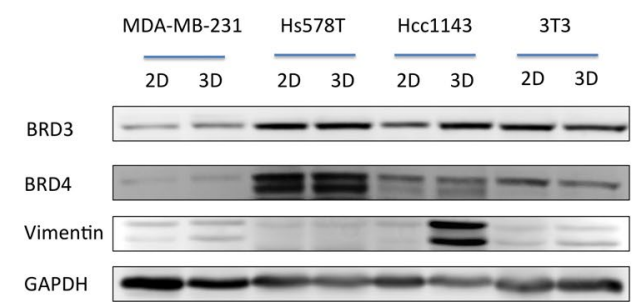

B
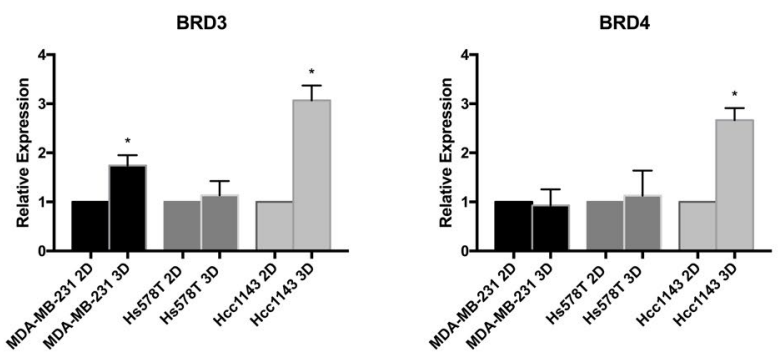

C
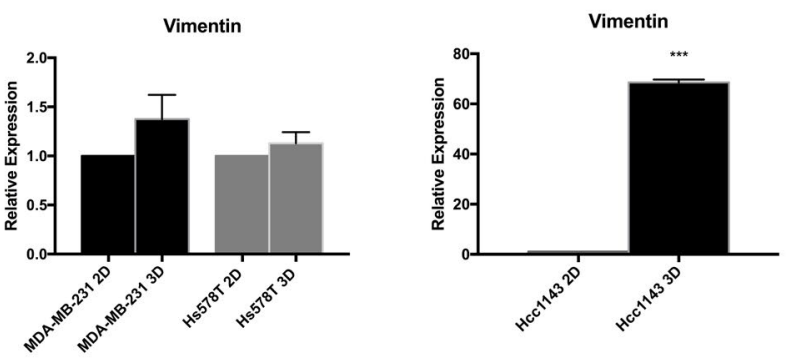

Figure 2. Brd3 and Brd4 were expressed in all TNBC cell lines in both $2 \mathrm{D}$ and $3 \mathrm{D}$ cultures and Vimentin was significantly induced in Hcc1143 cells in 3D.

A. Western blots show that $\mathrm{Brd} 3$ and $\mathrm{Brd} 44$ were expressed by TNBC cells in both 2D and 3D cultures at 48 hours. $3 T 3$ cells were used as a positive control. Significantly higher expression of Vimentin by Hcc1143 cells in 3D cultures suggests the presence of EMT.

B. Quantitation of the Western blot data shows increased Brd3 expression in MDA-MB-231 and Hcc1143 cells in 3D cultures and increased Brd4 expression in Hcc 1143 cells in $3 \mathrm{D}$ cultures relative to $2 \mathrm{D}$ cultures.

C. Vimentin expression was significantly increased in $\mathrm{Hcc} 1143$ in $3 \mathrm{D}$ cultures.

\section{EMT was induced in Hcc1143 cells in 3D cultures}

To detect the presence of epithelial-to-mesenchymal transition (EMT) Vimentin protein expression was examined. After 48 hours the basal-like Hcc1143 cells had 70 times higher vimentin expression in $3 \mathrm{D}$ culture than $2 \mathrm{D}$ cultures (Figure 2A, Figure 2C). This suggested that EMT occurred Hcc1143 cells when they were cultured in a 3D culture. However, there wasn't a significant change in vimentin expression for the mesenchymal-like MDA-MB-231 and Hs578T cell lines when they were cultured in 3D.

\section{JQ1 inhibited the growth of TNBCs}

JQ1 is a selective BET bromodomain (BRD) inhibitor that inhibits the expression of $\mathrm{Brd} 3$ and Brd4. MTT assays were performed to verify the JQ1 inhibition of TNBCs using carboplatin as positive control. Figures 3 and 4 show the cell viability of TNBCs with JQ1 and carboplatin treatment, respectively, in 2D. The $\mathrm{IC}_{50}$ of carboplatin for MDA-MB-231, $\mathrm{Hs} 578 \mathrm{~T}$ and $\mathrm{Hcc} 1143$ cells were $18.2 \mu \mathrm{M}, 5.3 \mu \mathrm{M}$ and $10.8 \mu \mathrm{M}$, respectively (Figure 3). This study found that the $\mathrm{IC}_{50}$ of JQ-1 for MDA-MB-231, Hs578T and Hcc1143 cells were $37.4 \pm 6.7$ $\mathrm{nM}, 113.5 \pm 8.7 \mathrm{nM}$ and $162.6 \pm 12.6 \mathrm{nM}$, respectively in the $2 \mathrm{D}$ culture system (Figure 4). All three cell lines were more sensitive to JQ-1 than to carboplatin in $2 \mathrm{D}$ cultures.

$3 \mathrm{D}$ viability assays determined the $\mathrm{IC}_{50}$ of JQ1 for MDAMB-231, Hs578T and Hcc1143 cells as $60.68 \mathrm{nM}, 81 \mathrm{nM}$ and $9.18 \mathrm{nM}$ in 3D cultures (Figure 5). Cell confluence in response to JQ1 treatment was assessed using an IncuCyte $\mathrm{ZOOM}^{\mathrm{TM}}$ Kinetic Imaging System. Figure 6 presents growth curves from the IncuCyte Zoom System showing the cell confluence of MDA-MB-231, Hs578T and Hcc1143 were all increasing slowly at lower doses of JQ1 $(<20 \mathrm{nM})$ and dramatically dropped at the higher concentrations of JQ1 (> $20 \mathrm{nM})$.

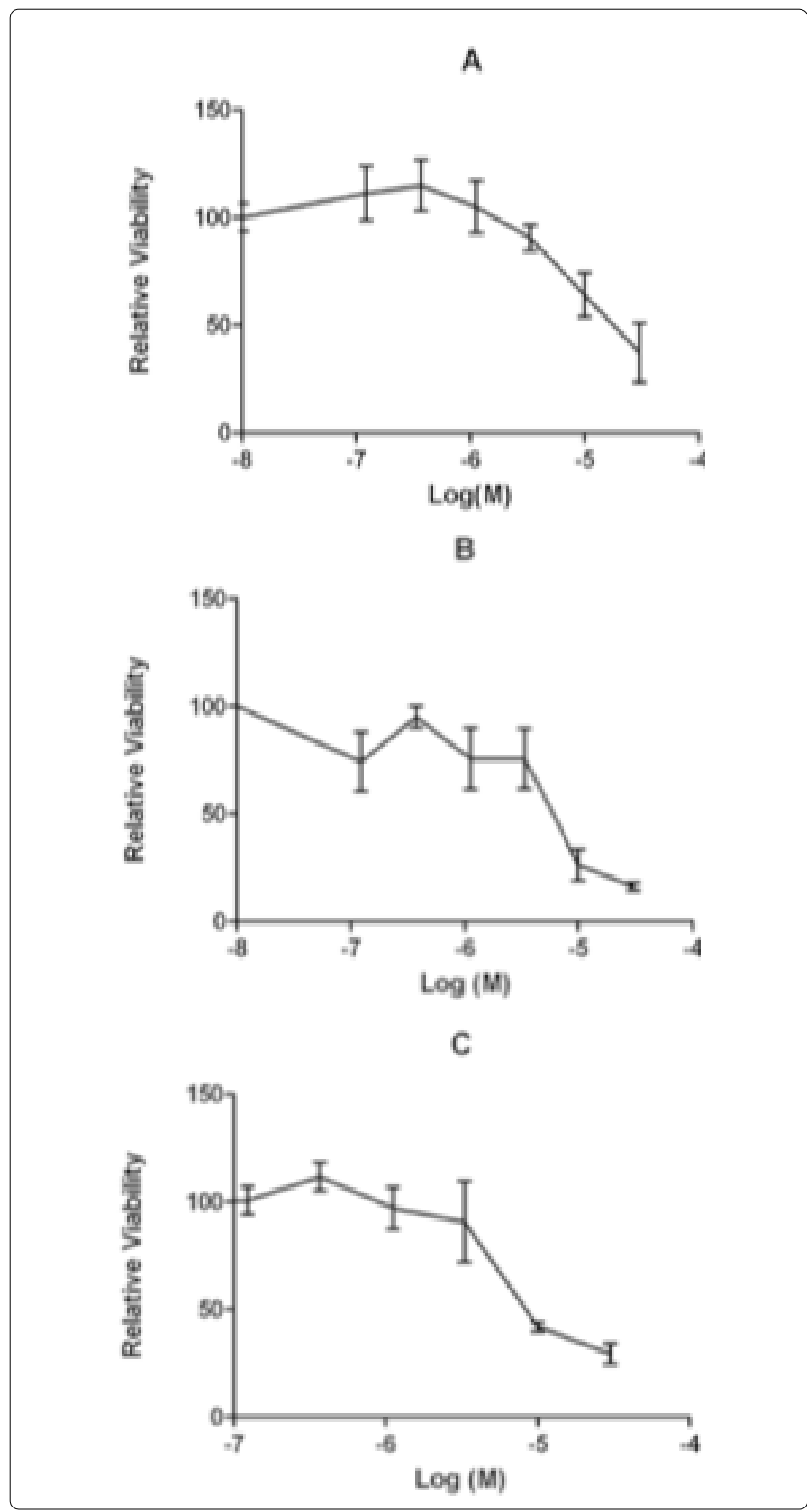

Figure 3. Carboplatin inhibited the growth of TNBC cells in 2D Cultures 
Carboplatin inhibition of A) MB-231, B) Hs578T, and C) Hcc1143 cells were determined by MTT Assay. Cells were treated with different doses of JQ1 for 3 days. Their $\mathrm{IC}_{50}$ were $18.2 \mu \mathrm{M}, 5.3 \mu \mathrm{M}$ and $10.8 \mu \mathrm{M}$, respectively.

\section{A}

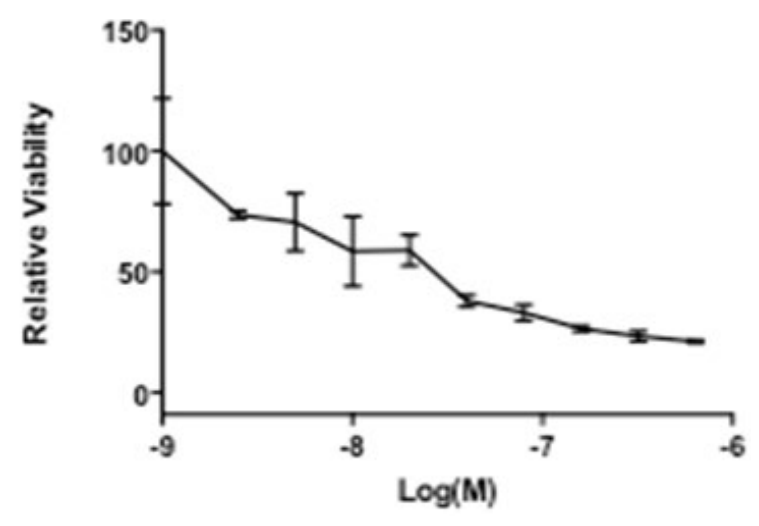

B
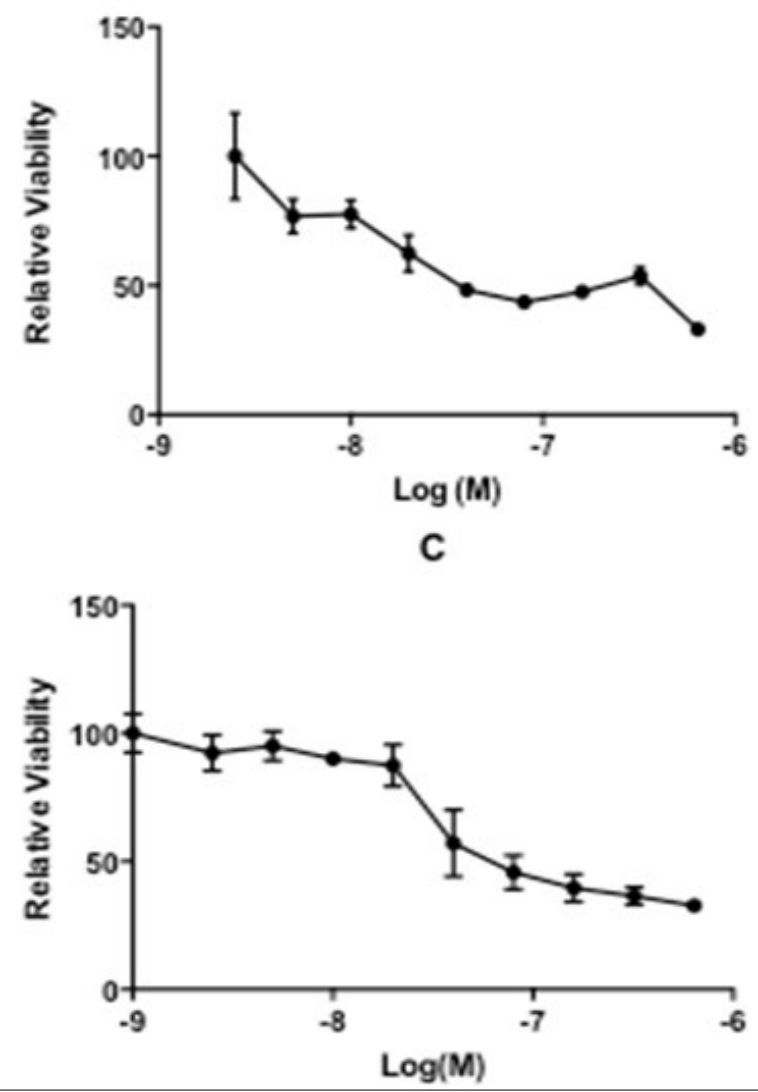

Figure 4. JQ1 inhibited the growth of TNBC cells in 2D cultures.

JQ1 inhibition of A) MB-231, B) Hs578T and C) Hcc1143 cells was determined by MTT assay. Cells were treated with different doses of JQ1 for 3 days. Their $I_{50}$ were $37.4 \pm 6.7$ $\mathrm{nM}, 113.5 \pm 8.7 \mathrm{nM}$ and162.6 $\pm 12.6 \mathrm{nM}$, respectively.
A

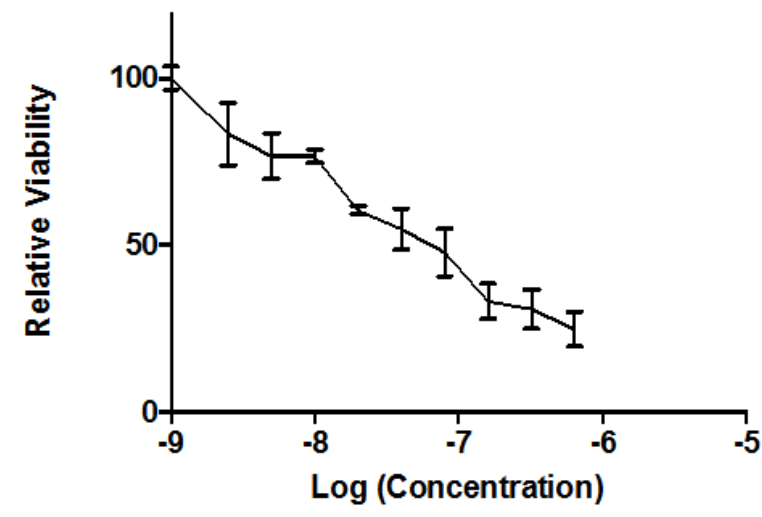

B
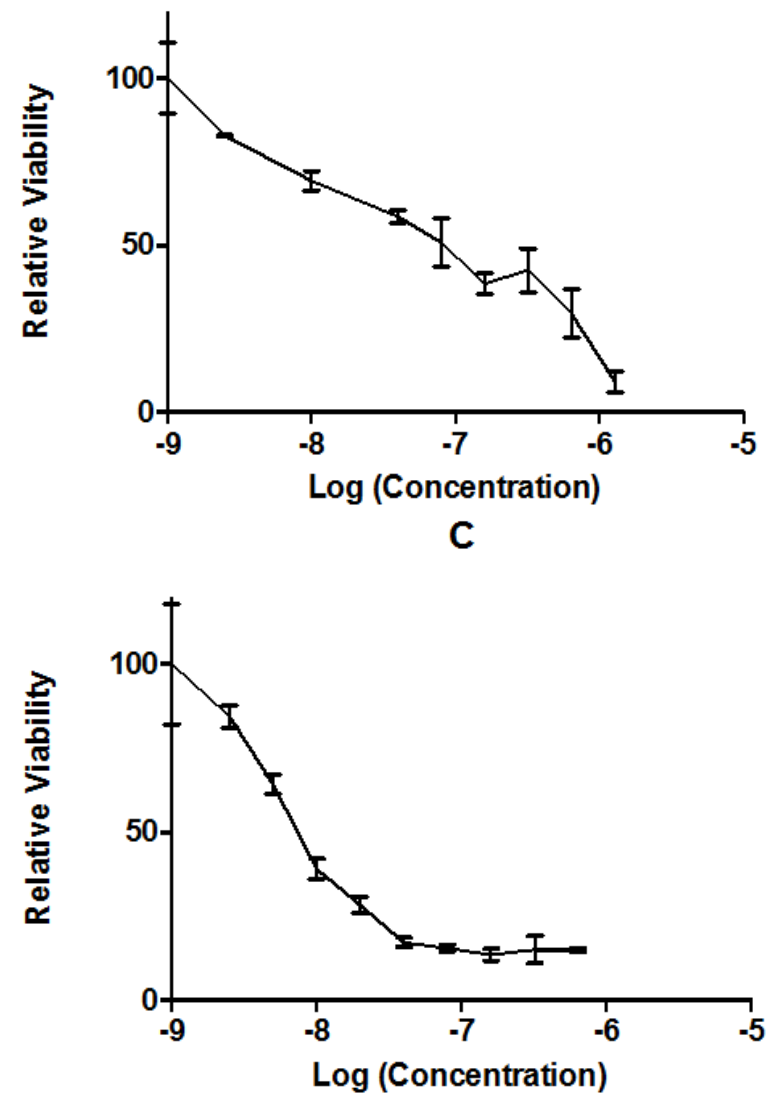

Figure 5. JQ1 inhibited the growth of TNBC cells in the 3D Culture System. The inhibition of JQ1 to A) MB-231, B) Hs578T and C) Hcc 1143 cells were determined by 3D Cell Viability Assay once when cells were treated with different doses of JQ1 for 3 days. Their $\mathrm{IC}_{50}$ were respectively $60.68 \mathrm{nM}, 81 \mathrm{nM}$ and $9.18 \mathrm{nM}$. 


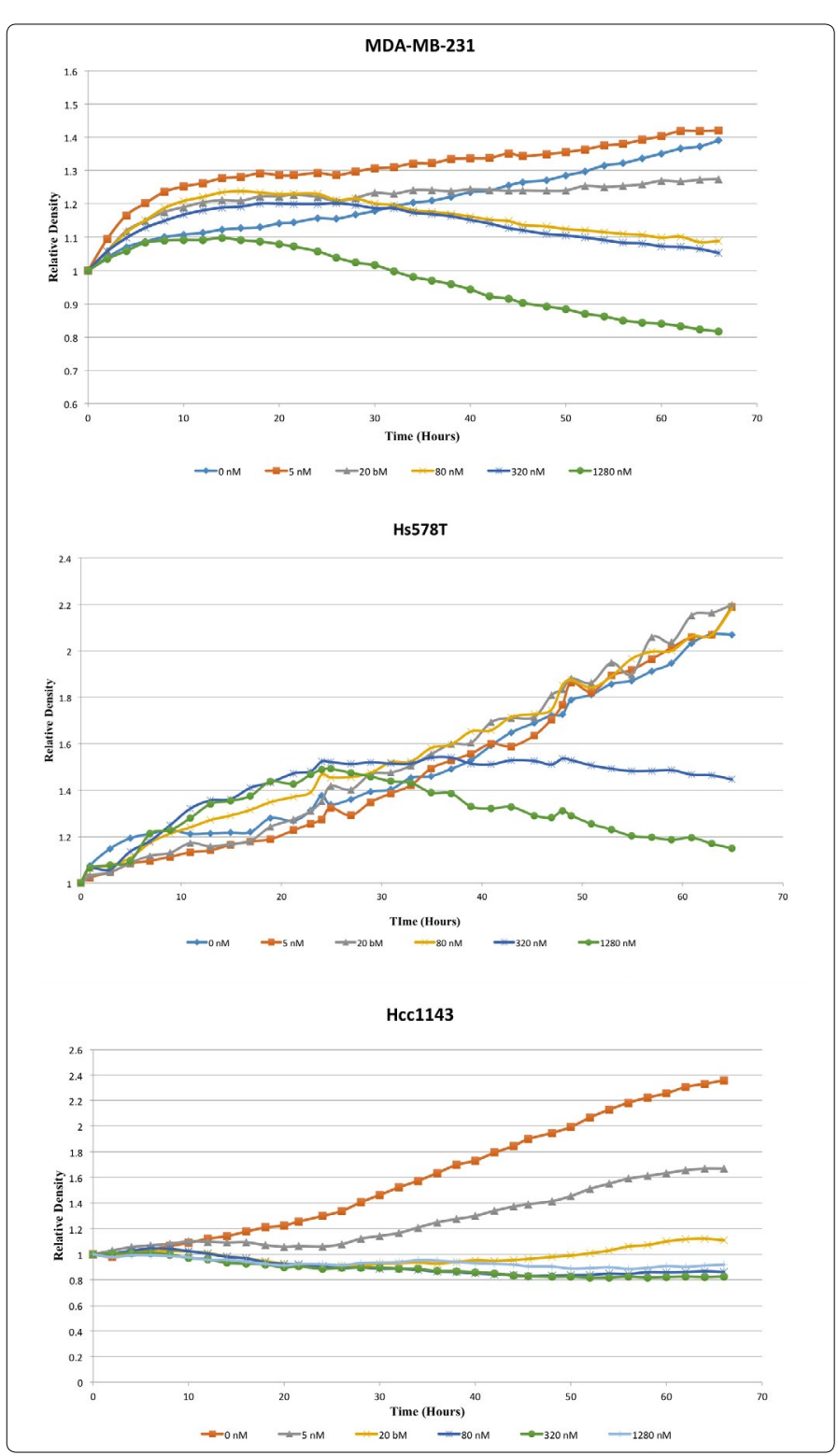

Figure 6. Growth of TNBC cells are JQ1 dose dependent in the 3D culture system. Growth curves were made by collecting confluence data every two hours over a 70 hour period in IncuCyte Zoom system. All three TNBC cells have nearly the same doubling time when they are treated with lower doses of JQ1 ( $<10 \mathrm{nM})$. TNBC cells stopped their growing after 24 hours of treatment with high doses of JQ1 (>20 nM).

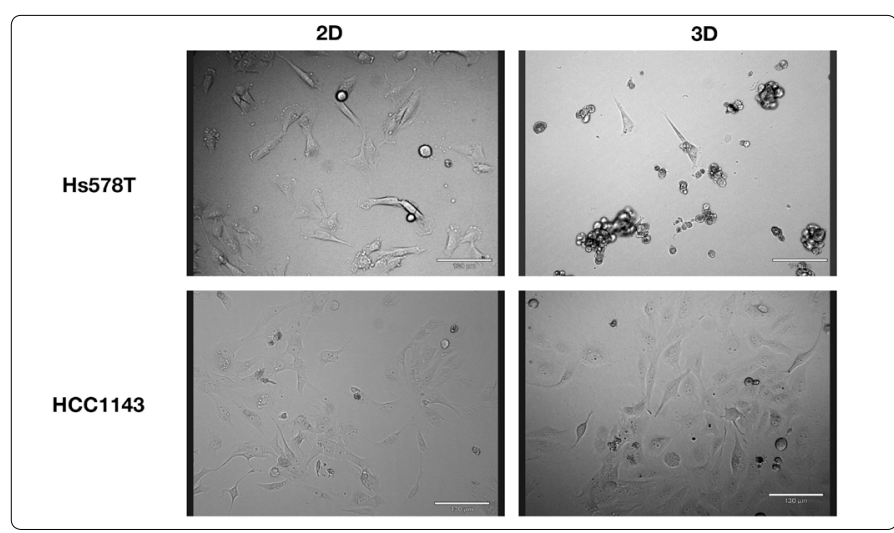

S1. Morphology changes of Hs578T cells in 3D cell culture system. Hs578T cells showed the morphology change when cultured in the 3D culture system, but HCC1143 didn't show the morphology change when cultured in the 3D culture system.

\section{Discussion}

Because of the lack of standard therapeutic targets in TNBCs, there is no effective receptor-based chemotherapy [2]. At present, the only drug approved by the FDA to treat TNBCs is carboplatin, which works by interfering with DNA repair [18]. However, the CALGB 40603 study (2014) concluded that additional treatment with carboplatin as the standard neo adjuvant chemotherapy after surgery for TNBCs increased the number of women who had a pathologically complete response [19]. Yet, the GeparSixto study (2014) acknowledged that the addition of carboplatin in the standard neo adjuvant chemotherapy for TNBCs improved disease-free survival from $76.1 \%$ to $85.5 \%$ [20]. Both studies unfortunately were not large enough to tell if adding carboplatin to the treatment of triple-negative breast cancer offered other benefits.

To model TNBCs, three different triple negative epithelial cells lines from breast carcinoma were used. MDA-MB-231 was derived from pleural effusion. Hs578T and Hcc1143 were derived from solid mammary gland tumors. The comparison of cells cultured in 2D and 3D systems showed differences in differentiation, gene expression, protein expression, and cell viability [21]. When Hs578T cells were cultured in the 3D culture system, the cells formed spheroid-like structures and clusters (S1). For HCC1143 cells, there was no morphology change when they were cultured in the 3D culture system (S1). The morphological changes in TNBC indicated that that Hs578T were mesenchymal-like, while HCC1143 cells were more basal-like TNBC [22].

Gene expression of BRD2, BRD3 and BRD4 was measured by $\mathrm{qPCR}$. We found that MDA-MB-231 and Hs578T had significantly higher BRD4 expression in 3D. Hcc1143, however, had significantly higher expression of all three genes in 3D. Protein expression of BRD3 and BRD4 was found in all three TNBC cell lines. Brd3 was found to have an $80 \%$ increase in MDA-MB-231 cells and a 200\% increase in Hcc 1143 cells in the 3D culture system. Brd4 was found to have a nearly $300 \%$ increase in Hcc1143 cells in the 3D culture system. All these increases make it probable that EMT or an increased migratory potential can induce Bromodomain expression in the 3D culture system, especially for basal-like cells.

The development of other cancers have been related to dysfunction of BET bromodomain proteins, and BRD inhibitors have shown efficacy in several cancer models [23]. JQ1 can displace BET bromodomain proteins, such as Brd4, from chromatin by competing with acetyl-lysine recognition modules leading to the inhibition of oncogenic transcriptional programs [24]. Indeed, JQ1 induced a dose-dependent decrease in cell viability. The inhibitory effect of JQ1 on TNBC cell viability had an $I C_{50}$ from $50 \mathrm{nM}$ to $120 \mathrm{nM}$ in the $2 \mathrm{D}$ cultures. Compared to carboplatin, JQ1 is about 1000 times more effective, which suggests that JQ1 would a potential effective therapy for TNBC. The IC $C_{50}$ of JQ1 for TNBCs in the $3 \mathrm{D}$ cultures was about $50 \mathrm{nM}$, demonstrating the effectiveness of JQ1 in the 3D cultures. IC $C_{50}$ of JQ1 to Hcc1143 decreased from $160 \mathrm{nM}$ in $2 \mathrm{D}$ to $9 \mathrm{nM}$ in $3 \mathrm{D}$, indicating that Hcc1143 is more sensitive to JQ1 in the 3D cultures. This is interesting as 
the Hcc1143 cells has the most extensive shift in vimentin expression when moved to 3D cultures. It is also possible that the presence of EMT in 3D cultures increases the sensitivity to JQ1. Vimentin, related to EMT in breast cancer, can therefore be used as a biomarker for this sensitivity.

JQ1 binds to acetylated lysines blocking BET protein binding, but the function of JQ1 lacks selectivity within the BET family. This makes it difficult to clarify contributions of each protein family member to transcriptional and cellular outcomes [25]. The detailed pathways related to BRDs on TNBCs may still be unknown, but some promising findings resulted in an effect of JQ1 on the BRDs pathway and the relationship among Brd2, Brd3 and Brd4.

Hnilicová (2013) reported that the C-terminal domains of $\mathrm{Brd} 2$ were associated with chromatin modifying gene splicing, and Brd4 stimulated constitutive splicing of primary response genes likely via modulating RNA polymerase II C-terminus phosphorylation [26]. The knockdown of Brd2 was shown to result in reduced expression of almost half of alternative splicing targets because Brd2 influenced the formation of the initiation complex at the promoter [26]. The genes of the promoter stimulated transcription and regulated alternative splicing at the same time. However, the molecular mechanism of $\mathrm{Brd} 2$ mediated splicing regulation is also unknown.

It is also worth mentioning that expression of cell cycle regulators p21 and p53 have been found related to BRD2 expression. p21 transcription decreased $\sim 60 \%$ and p53 increased $\sim 60 \%$ after the knockdown of BRD2 [26]. It's likely that JQ1 inhibits the expression of Brd2 which then effects the transcription of at least 1400 genes, causing the termination of cell communication, cell differentiation, regulation of cell adhesion and macromolecule modification [26]. JQ1 was found to inhibit the expression of Brd4, a dynamic regulator of breast cancer metastasis through modulation of the extracellular matrix [23]. Brd4 binds to the c-MYC promoter, an action that is reversed by JQ1.

Studies have shown that Brd4 inhibition leads to cell cycle arrest, senescence, p21 upregulation and apoptosis, all processes mediated by $\mathrm{p} 53$, a requirement for c-MYCdependent cell cycle arrest and differentiation, but not apoptosis [27-30]. Still, JQ1 does not exhibit a direct effect of Brd4 inhibition on p53 protein levels [10,27,31]. A finding consistent with the actions of JQ1 where ectopic expression of c-MYC in hematological cell lines confers significant resistance to JQ1-induced cell cycle arrest and differentiation, but cell death is not affected [27]. It has also been determined that JQ1 cooperatively removes Brd4 from chromatin and increases re-assembly of the 7SK snRNP, which can silence the transcription of c-Myc, resulting in growth arrest and apoptosis of multiple myeloma cells [32]. Synergistic effects of histone deacetylases and JQ1 could possibly be exploited for better treatment of these conditions and for future interventions for TNBCs.

In the other research, it was also determined that after the knockdown of BRD2, the transcription of BRD4 declined by almost $75 \%$, whereas BRD3 expression decreased by $\sim 40 \%$ which suggest possible cross-regulation of BET proteins [26]. Most likely, the 3D cell culture model will have different effect on the expression of $\mathrm{Brd} 2$ in different cell lines. The coregulation among $\mathrm{Brd} 2, \mathrm{Brd} 3$ and $\mathrm{Brd} 4$ could result in different expression of BRDs in different cell lines. Brd2 and $\mathrm{Brd} 3$ associated chromatin is significantly enriched in $\mathrm{H} 4 \mathrm{~K} 5, \mathrm{H} 4 \mathrm{~K} 12$ and H3K14 acetylation and contains relatively little dimethylated H3K9 [33]. Several studies focused on BRD2 and BRD4, but little is known about the properties of Brd3. LeRoy et al. (2008), found that $\mathrm{Brd} 2$ and $\mathrm{Brd} 3$ bind actively transcribed, acetylated chromatin along the cyclin D1 gene $[33,34]$. They also determined that Brd2 and Brd3 facilitate RNA polymerase II transcription through acetylated nucleosomes $[33,35]$.

Our study found that $\mathrm{Brd} 3$ and $\mathrm{Brd} 4$ transcripts are present and the proteins expressed in TNBC cells. In our 3D culture system, cells were in a mesenchymal state, which induced the expression of bromodomain proteins. With the higher expression of BRDs, TNBC cells were more sensitive to the JQ1. JQ1 can inhibit the growth of TNBCs, but the underlying pathway was not studied here. However, based on the other studies, we propose the potential pathway would be Brd2-p21/p53 pathway and Brd4-cMYC pathway $[36,10,26,27]$. It has been shown that MYC can induced EMT in breast cancer cells [30], and Hcc1143 cells in 3D cell culture system have been shown in the transition from epithelial to mesenchymal, which suggests that $\mathrm{HCC} 1143$ cells have higher MYC expression in 3D cell culture system. JQ1 is BET inhibition and targets MYC dependent in cancer cells [31]. The overexpression of MYC induces the expression of BRD4, and EMT also recruits BRD4 to direct WNT5A expression in basal-like breast cancer [32]. BRD4 induction caused by MYC and EMT make HCC1143 cells more sensitive to JQ1 in 3D cell culture system.

\section{Conclusion}

JQ1 inhibits the growth of TNBC cells in both 2D and 3D cell culture systems. Our data suggests that cells that are more mobile have an increased sensitivity to bromodomain inhibitors. This provides a possible target for early metastasis of triple negative breast cancers.

\section{Acknowledgment}

We extend our gratitude to Dr. Christina Zito and Dr. Eva Sapi for the great support and suggestions on this study, and we also thank Dr. Gil Mor for the use of the IncuCyte Zoom.

\section{References}

1. Siegel RL, Miller KD, Jemal A. Cancer statistics. CA Cancer J Clin. 2017; 67(1): 7-30. doi: 10.3322/caac.21387

2. Ismail-Khan R, Bui MM. A review of triple-negative breast cancer. Cancer Control. 2010; 17(3): 173-176.

3. American Cancer Society. Cancer Facts \& Figures 2017

4. Knapp S. Discovery of BET bromodomain inhibitors and their role in target validation. Med Chem Commun. 2013; 5: 288-296. doi: 10.1039/ C3MD00291H 
5. Zeng $L$, Zhou MM. Bromodomain: an acetyl-lysine binding domain. FEBS Lett. 2002; 513(1): 124-128.

6. Baumli S, Lolli G, Lowe ED. The structure of P-TEFb (CDK9/cyclin T1), its complex with flavopiridol and regulation by phosphorylation. EMBO J. 2008; 27(13): 1907-1918. doi: 10.1038/emboj.2008.121

7. Barbieri I, Cannizzaro E, Dawson MA. Bromodomains as therapeutic targets in cancer. Brief Funct Genomics. 2013; 12(3): 219-230. doi: 10.1093/bfgp/elt007

8. Garcia-Gutierrez P, Mundi M, Garcia-Dominguez M. Association of bromodomain BET proteins with chromatin requires dimerization through the conserved motif B. J Cell Sci. 2012; 125(Pt 15): 3671-3680. doi: 10.1242/ jcs. 105841

9. Filippakopoulos P, Qi J, Picaud S. Selective inhibition of BET bromodomains. Nature. 2011; 468(7327): 1067-1073. doi: 10.1038/nature09504.Selectiv.

10. Delmore JE, Issa GC, Lemieux ME. BET bromodomain inhibition as a therapeutic strategy to target c-Myc. Cell. 2011; 146(6): 904-917. doi: 10.1016/j.cell.2011.08.017

11. Cheng $Z$, Gong Y, Ma Y. Inhibition of BET Bromodomain Targets Genetically Diverse Glioblastoma. 2013; 19(7): 1748-59. doi: 10.1158/10780432.CCR-12-3066

12. Zuber J, Shi J, Wang E. RNAi screen identifies Brd4 as a therapeutic target in acute myeloid leukaemia. Nature. 2011; 478(7370): 524-528. doi: 10.1038/nature10334

13. Lee J, Cuddihy MJ, Kotov N . Three-dimensional cell culture matrices: state of the art. Tissue Eng Part B Rev. 2008; 14(1): 61-86. doi: 10.1089/ teb.2007.0150

14. Prestwich GD. Simplifying the extracellular matrix for 3-D cell culture and tissue engineering: A pragmatic approach. J Cell Biochem. 2007; 101(6): 1370-1383. doi: 10.1002/jcb.21386

15. Tsai JH, Yang J. Epithelial - mesenchymal plasticity in carcinoma metastasis. Genes Dev. 2013; 27(20): 2192-2206. doi: 10.1101/gad.225334.113.2192

16. Sarrió D, Rodriguez-Pinilla SM, Hardisson D, Cano A, Moreno-Bueno G, et al. Epithelial-mesenchymal transition in breast cancer relates to the basallike phenotype. Cancer Res. 2008; 68(4): 989-997. doi: 10.1158/0008-5472. CAN-07-2017

17. Baum B, Settleman J, Quinlan MP. Transitions between epithelial and mesenchymal states in development and disease. Semin Cell Dev Biol. 2008; 19(3): 294-308. doi: 10.1016/j.semcdb.2008.02.001

18. Monneret $C$. Platinum anticancer drugs. From serendipity to rational design. Ann Pharm Fr. 2011; 69(6): 286-295. doi: 10.1016/j.pharma.2011.10.001

19. Sikov WM, Hospital M. Impact of the Addition of Carboplatin and / or Bevacizumab to Neoadjuvant Once-per-Week Paclitaxel Followed by DoseDense Doxorubicin and Cyclophosphamide on Pathologic Complete Response Rates in Stage II to III Triple-Negative Breast Cancer : CALGB 40603. J Clin Oncol. 2014; 33(1): 13-21. doi: 10.1200/JCO.2014.57.0572

20. Von Minckwitz G, Schneeweiss A, Loibl S. Neoadjuvant carboplatin in patients with triple-negative and HER2-positive early breast cancer (GeparSixto; GBG 66): A randomised phase 2 trial. Lancet Oncol. 2014; 15(7): 747-756. doi: 10.1016/S1470-2045(14)70160-3.

21. Anton $D$, Burckel $H$, Josset $E$, Noel $G$. Three-dimensional cell culture: $A$ breakthrough in vivo. Int J Mol Sci. 2015; 16(3): 5517-5527. doi: 10.3390/ ijms 16035517
22. Chavez KJ, Garimella SV, Lipkowitz S. Triple negative breast cancer cell lines: One tool in the search for better treatment of triple negative breast cancer. Breast Dis. 2010; 32(1-2): 35-48. doi: 10.3233/BD-2010-0307

23. Alsarraj J, Hunter KW. Bromodomain-Containing Protein 4: A Dynamic Regulator of Breast Cancer Metastasis through Modulation of the Extracellular Matrix. Int J Breast Cancer. 2012; 2012: 670632. doi: $10.1155 / 2012 / 670632$

24. Shu $\mathrm{S}$, Lin $\mathrm{CY}, \mathrm{He} H \mathrm{H}$. Response and resistance to BET bromodomain inhibitors in triple-negative breast cancer. Nature. 2016; 529(7586): 1-24. doi: $10.1038 /$ nature 16508

25. Deeney JT, Belkina AC, Shirihai OS, Corkey BE, Denis G V. BET Bromodomain proteins $\mathrm{Brd} 2, \mathrm{Brd} 3$ and $\mathrm{Brd} 4$ selectively regulate metabolic pathways in the pancreatic $\beta$-cell. PLoS One. 2016; 11(3). doi: 10.1371/journal. pone. 0151329

26. Hnilicová J, Hozeifi S, Stejskalová E. The C-terminal domain of Brd2 is important for chromatin interaction and regulation of transcription and alternative splicing. Mol Biol Cell. 2013; 24(22): 3557-3568. doi: 10.1091/ mbc.E13-06-0303

27. Stewart HJS, Horne GA, Bastow S, Chevassut TJT. BRD4 associates with p53 in DNMT3A-mutated leukemia cells and is implicated in apoptosis by the bromodomain inhibitor JQ1. Cancer Med. 2013; 2(6): 826-835. doi: $10.1002 / \mathrm{cam} 4.146$

28. Acosta JC, Gil J. Senescence: A new weapon for cancer therapy. Trends Cell Biol. 2012; 22(4): 211-219. doi: 10.1016/j.tcb.2011.11.006

29. Ho JSL, Ma W, Mao DYL, Benchimol S. p53-Dependent transcriptional repression of c-myc is required for G1 cell cycle arrest. Mol Cell Biol. 2005; 25(17): 7423-7431. doi: 10.1128/MCB.25.17.7423-7431.2005

30. Wu SY, Lee AY, Lai HT, Zhang H, Chiang CM. Phospho switch triggers brd4 chromatin binding and activator recruitment for gene-specific targeting. Mol Cell. 2013; 49(5): 843-857. doi: 10.1016/j.molcel.2012.12.006

31. Li Z, Guo J, Wu Y, Zhou Q. The BET bromodomain inhibitor JQ1 activates HIV latency through antagonizing Brd4 inhibition of Tat-transactivation. Nucleic Acids Res. 2013; 41(1): 277-287. doi: 10.1093/nar/gks976

32. Bartholomeeusen $\mathrm{K}$, Xiang $\mathrm{Y}$, Fujinaga $\mathrm{K}$, Peterlin BM. Bromodomain and extra-terminal (BET) bromodomain inhibition activate transcription via transient release of Positive Transcription Elongation Factor $\mathrm{b}(\mathrm{P}-\mathrm{TEFb})$ from 7SK small nuclear ribonucleoprotein. J Biol Chem. 2012; 287(43): 36609-36616. doi: 10.1074/jbc.M112.410746.

33. LeRoy G, Rickards B, Flint SJ. The Double Bromodomain Proteins Brd2 and Brd3 Couple Histone Acetylation to Transcription. Mol Cell. 2008; 30(1): 51-60. doi: $10.1016 /$ j.molcel.2008.01.018

34. Sinha A, Faller DV, Denis GV. Bromodomain analysis of Brd2-dependent transcriptional activation of cyclin A. Biochem J. 2005; 387(Pt 1): 257-269. doi: 10.1042/BJ20041793

35. Cheung KL, Zhang F, Jaganathan A. Distinct Roles of Brd2 and Brd4 in Potentiating the Transcriptional Program for Th17 Cell Differentiation. Mol Cell. 2017; 65(6): 1068-1080. doi: 10.1016/j.molcel.2016.12.022

36. Mertz JA, Conery AR, Bryant BM. Targeting MYC dependence in cancer by inhibiting BET bromodomains. Proc Natl Acad Sci USA. 2011; 108: 1666916674. doi: $10.1073 /$ pnas. 1108190108. 